\title{
Breathe
}

\section{Pleuritic chest pain in a young asylum seeker}

\section{Case report}

A 17-year-old asylum seeker from Afghanistan presented to the emergency department with chest pain. He had travelled to the UK after residing in multiple European cities. He presented with a 1-week history of right-sided pleuritic chest pain. There was no evidence of a productive cough or recent viral illness and he had no history of injury to the chest. In addition to his chest pain, he had been suffering from persistent right upper quadrant discomfort for approximately 3 years. There was no nausea or vomiting and he denied any bowel or urinary symptoms. He had experienced episodes of fever and loss of appetite but no change in weight. His past medical background consisted of black water fever, as a consequence of malaria, and a previous gunshot wound, which had now healed. He took no regular medications and all of his clinical observations were stable at the time of presentation.

On examination, decreased breath sounds and dullness to percussion were detected over the right lung base. All blood results, including eosinophil count and liver function tests, were normal. The admission chest radiograph revealed what appeared to be a cystic lesion within the right lower lung zone (figure 1).

Echinococcus serology was requested alongside a CT scan of the thorax, abdomen and pelvis. The

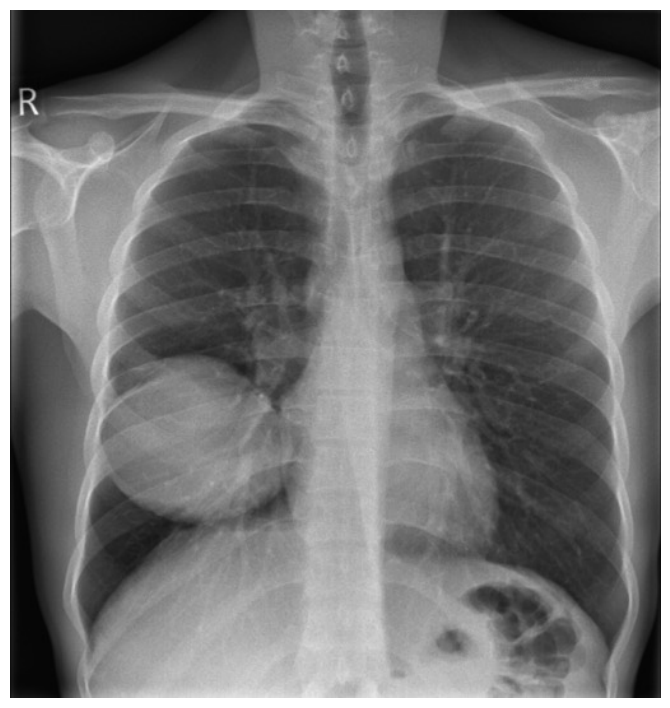

Figure 1 Admission chest radiograph demonstrating a sharply circumscribed, large fluid density within the right lower zone. Hilar overlay and preservation of the right heart border suggests it to be in the posterior chest. Homogenous density and very sharp margins make a cyst more likely than a mass.

\section{Task 1}

Based on the history and chest radiograph, which of the following differential diagnoses is the most likely?
a) Lung malignancy
b) Hydatid cyst (Echinococcus)
c) Foreign body
d) Pneumonia

Cite as: Zahedi D, Moori P, Ashraf I, et al. Pleuritic chest pain in a young asylum seeker Breathe 2020; 16: 190294. 


\section{Answer 1}

b) The chest radiograph has classical features of a cystic lesion within the lung. A cystic airspace can be visualised as a well-defined, thin walled parenchymal space often with an air fluid level. In malignancy, lesions are denser and less defined. Pneumonia usually appears as ill-defined patchy areas of increased density and consolidation.

\section{Task 2}

What would be the next appropriate step to further investigate the chest radiograph findings?
a) Bronchoscopy and pleural biopsy
b) Computed tomography (CT)
c) Lung function tests
d) Pleural ultrasound scan

Echinococcus serology returned as negative. The CT scan showed a well-defined, thick walled cystic area in the right mid to lower lung zone and no other abnormalities were noted (figure 2). The radiological findings together with the patients' travel history most likely represented hydatid disease. The Infectious Diseases Department at the Royal Liverpool University Hospital (Liverpool, UK) were contacted, who advised that after discussion within their multidisciplinary team, this patient would benefit from a lobectomy.

\section{Answer 2}

b) CT of the thorax and abdomen would be useful in the next stages. The scan would help to better visualise the lung parenchyma and mediastinum, in addition to assessing the anatomical involvement of the identified pathology. This would help to further assess its density and create an appropriate management plan.

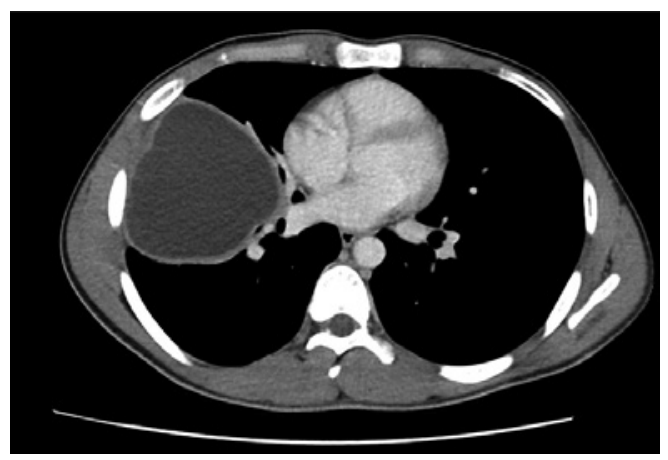

Figure 2 Computed tomography thorax slice. Right mid and lower lung zone well-defined thick walled fluid density/ cystic area measuring $\otimes 9 \times 7 \mathrm{~cm}$ representing a cyst. Solid abdominal organs appear unremarkable.

\section{Task 3}

What is the most common definitive host of the Echinococcus parasite?
a) Cats
b) Dogs
c) Rats
d) Rabbits 


\section{Answer 3}

b) Dogs are the most common definitive hosts of the Echinococcus parasite, they usually carry the parasite within their intestines.

3 weeks post-discharge the individual was re-admitted due to a 4-day history of a productive cough with yellow sputum, in addition to vomiting, fevers and generalised abdominal pain. A chest radiograph was arranged, which revealed a change in appearance of the lung cyst (figure 3). The new features were suggestive of a ruptured cyst. The patient was commenced on praziquantel and a repeat CT scan of the thorax was performed.

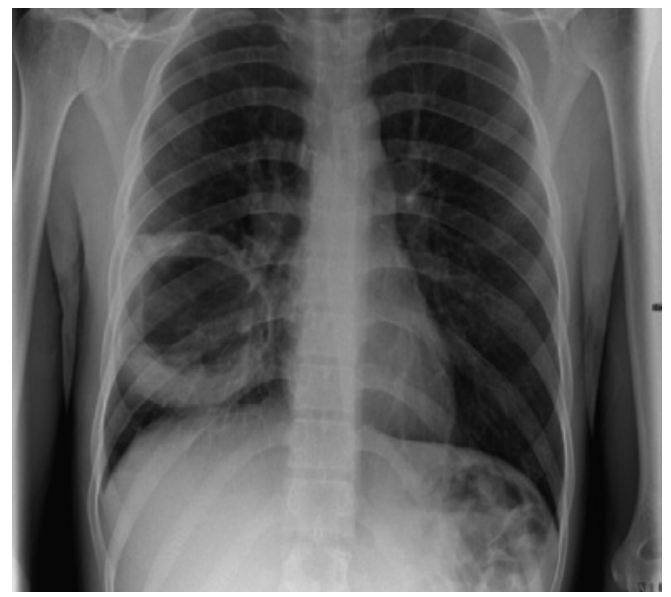

Figure 3 Re-admission chest radiograph. The cyst in the right lower lobe now demonstrates central lucency with a thickened wall. No focal consolidation and no pleural effusion.

\section{Task 4}

Describe the CT image in figure 4.

\section{Answer 4}

The CT image showed the "water lily" sign that is indicative of cyst rupture (figure 4), which changed the diagnosis from a simple to a complicated hydatid cyst.

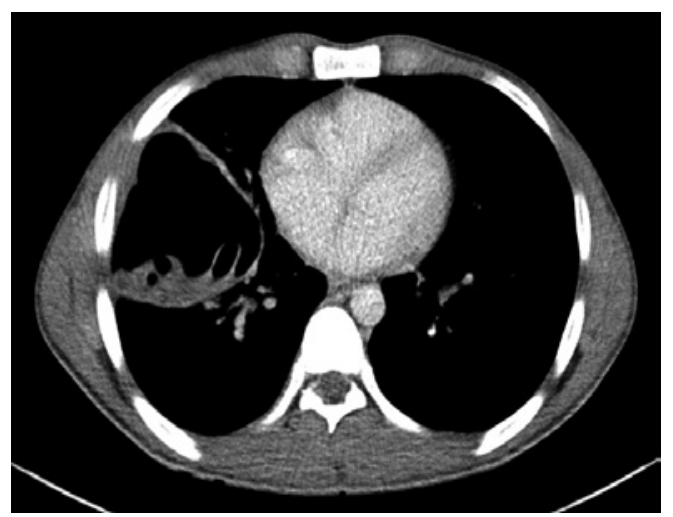

Figure 4 Repeat computed tomography. Since the previous scan 6 weeks ago the endocyst has detached and is laying dependently within a now predominantly air filled pericyst (water lily sign).

Due to these findings, the patient was commenced on albendazole alongside praziquantel and discharged awaiting a lobectomy. Sputum results from the second admission demonstrated presence of Echinococcus.

\section{Task 5}

What is the mechanism of action of albendazole?

a) It inhibits the cytochrome P450 enzyme 14- $\alpha$-demethylase

b) It binds to colchicine-sensitive sites of $\beta$-tubulin

c) It binds to dihydrofolate reductase thus inhibiting the reduction of dihydrofolic acid to tetrahydrofolic acid

d) It inhibits the transduction of DNA gyrase 


\section{Answer 5}

b) Albendazole creates degenerative changes by binding to colchicine-sensitive sites of $\beta$-tubuline within worm intestines, inhibiting microtubule formation. Albendazole impairs glucose uptake, depleting glycogen stores in larval stages. Egg production is blocked by preventing spindle formation, essential for cell division. At increased concentrations, the metabolic pathways of helminths are disrupted by inhibiting enzymes maleate dehydrogenase and fumarate reductase. This in turn reduces ATP production, resulting in parasitic death.

The patient underwent a right thoracotomy and excision of hydatid cyst, capitonnage and sublobar resection. The excised tissue was examined histologically. A ruptured cyst wall measuring $100 \times 75 \mathrm{~mm}$ with the wall measuring $1 \mathrm{~mm}$ and four pieces of disrupted fibrotic possible lung tissue measuring $125 \times 90 \times 12 \mathrm{~mm}$ and weighing $55 \mathrm{~g}$ were examined microscopically. The sections showed a lobulated cyst wall with evidence of degenerative projections forming daughter cysts and areas of sediment within. There was evidence of a fibrotic chronic inflammatory process noted in the lung tissue but importantly, no evidence of malignancy. These features were consistent with the clinical impression of hydatid cyst disease and confirmed the diagnosis. The patient was reviewed in the outpatient clinic 8 weeks post-operatively. He had recovered well from the surgery and completed his anti-helmintic medication course. A follow up chest radiograph showed post-operative changes with a loculated right pneumothorax and loss of volume in the right lung (figure 5). The patient was otherwise clinically stable and asymptomatic. He remains under follow-up at present.

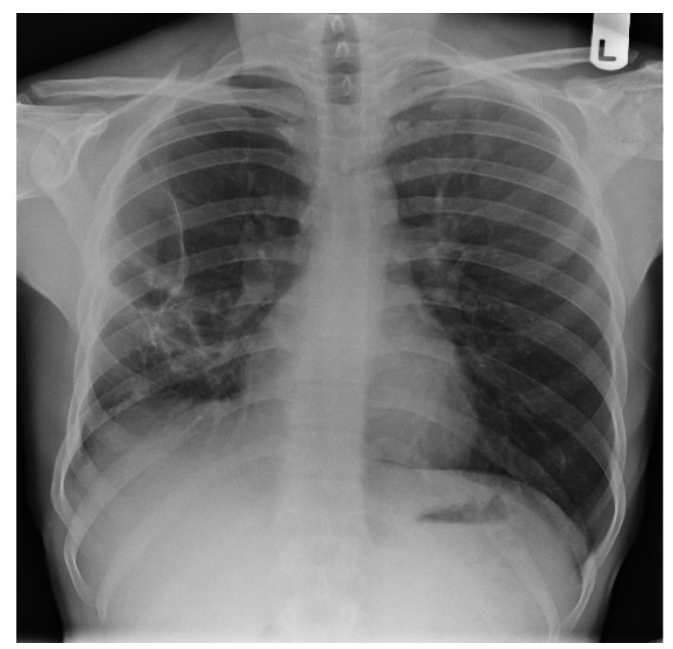

Figure 5 Post-operative chest radiograph demonstrating a loculated right-sided pneumothorax with loss of volume of the right lung.

\section{Discussion}

\section{Life cycle and clinical presentation}

Hydatid disease, also known as Echinococcosis, is caused by the tapeworm larvae Echinococcus. Contamination with Echinococcus granulosus is the most common form of infection in humans [1]. Since 2000, there have been 77 reported cases in England and 19 reported cases in Wales. Infestation with the Echinococcus larvae may result in cyst formation throughout any organ within the body. Hydatid cyst development most commonly occurs within the liver and pulmonary structures [2, 3]. Other areas of the body are less commonly affected; however, cases have been reported of cyst formation within muscle groups [4]. Primary pulmonary Echinococcosis without liver involvement is an uncommon phenomenon [5, 6]. Hippocrates first defined Echinococcosis in a human liver as "cysts full of water" and approximately 1000 years ago, the famous physician Al-Rahzes recognised and documented hydatid disease of the liver [7]. The manifestation of pulmonary hydatidosis can have varied clinical presentations. Symptoms such as cough, haemoptysis, dyspnoea and chest pain have all been reported [8]. Spontaneous rupture of hydatid cysts into a bronchus may occur, however accidental rupture through aspiration is more commonly seen. This case demonstrates primary pulmonary hydatidosis with spontaneous rupture into the bronchus. This case illustrates the challenges of diagnosing pulmonary hydatid disease and the associated complications that may result from delay in diagnosis.

The Echinococcus parasite comprises of a definitive host, usually dogs, and an intermediate host, usually sheep. Within the definitive canine host, the tapeworm and its larvae reside within the intestines [9]. Once the eggs, also known as oncospheres, are released, they are then excreted into the faeces. Once ingested, these eggs cause infections by hatching into larvae and form swellings, which are known as hydatid cysts [10]. The eggs are able to penetrate the intestines, entering the portal circulation and residing within the liver. Oncospheres are not always filtered by the liver and can remain within the pulmonary vasculature $[8,11]$. Humans along with sheep act as the intermediate host and can become infected by ingesting tapeworm eggs from infected canine hosts. The eggs can also cause primary lung disease if inhaled [12]. Symptoms of the disease may take years to develop and depend on the location of the cyst; cysts in the liver produce jaundice and abdominal pain while cysts in the lung cause breathlessness, cough and chest pain. Occasionally these cysts can rupture, resulting in pyrexia and an anaphylaxis-type reaction [13]. This case represents primary pulmonary hydatidosis, suggesting possible inhalation of the oncospheres. Cyst rupture is the most common complication of symptomatic 
primary pulmonary hydatidosis. Rupture may be as a consequence of trauma or secondary infection and patients will often present with fever and cough with expectoration of clear fluid [1].

\section{Echinococcus serology and imaging}

Serological methods such as ELISA, indirect haemagluttination (IHA) test, latex agglutination test and immunoblotting tests are used in the detection of hydatid cyst disease. The ELISA uses antibodies to detect Echinococcus granulosusspecific antigens, resulting in colour changes if these antigens are present $[14,15]$. Echinococcus serology using the ELISA method is $97 \%$ sensitive. A negative Echinococcus serology often occurs in cases of proven extra-hepatic disease and one in two cases of hydatid disease will have negative serology $[16,17]$. This was true for the case described and is an important point to be noted when investigating this disease form. The second sputum was likely positive due to subsequent rupture of the cyst into a neighbouring bronchus and expulsion within the saliva. The IHA test looks for agglutination causes by antigen-antibody pairs, with the clumping of latex beads coated with antigens and antibodies showing the presence of these pairs in the latex agglutination test [18]. Ultrasound imaging is the gold standard investigation, in combination with $\mathrm{CT}$ or magnetic resonance imaging (MRI) [19]. Ultrasound allows classification of the cysts and is a useful tool for initial investigation; however, MRI can be useful when assessing the anatomical relation of cysts, assisting in surgical planning [20]. Diagnosis of Echinococcus relies on a combination of clinical information relating to the history of exposure alongside the clinical presentation, serology and radiological imaging.

\section{Treating hydatid disease}

Treatment of pulmonary hydatid disease is predominantly surgical, especially in the case of complicated or large cysts [21]. Patients who are not suitable for surgical intervention may be treated with anti-helmintics for many years. Other management approaches include percutaneous sterilisation with the PAIR technique (puncture, aspiration, injection, re-aspiration) and watchful waiting. Surgery is curative but there is variable evidence regarding the mortality, morbidity and relapse rates [2, 13]. Surgical options available for the management of pulmonary cysts include lobectomy, wedge resection, peri-cystectomy, intact peri-cystectomy and capitonnage. Adjuvant medical therapy is administered to minimise the risk of secondary Echinococcosis, which may occur through the process of seeding if fluid spillage of the cyst contents occurs in the thoracic cavity [22, 23]. This may also reduce the risk of recurrence and facilitate removal of the cyst by reducing intracystic pressure. Dissemination of cysts has been found to be higher risk in larger cysts. In terms of medical therapy, there are only two antiparasitic medications that are proven to be effective against cystic Echinococcus, these are albendazole and mebendazole [24]. Some practioners use praziquantel in addition to albendazole although there is no clear guidance regarding its efficacy [25]. Albendazole is the drug of choice due to its higher rate of systemic absorption and penetration into hydatid cysts as compared with mebendazole [26]. Albendazole is usually commenced 1 week prior to surgery and continued for a minimum of 4 weeks post-operatively.

\section{Conclusion}

Hydatid disease predominantly affects the hepatic and pulmonary body systems and pulmonary hydatidosis may present with a range of clinical features. In this case of primary pulmonary hydatidosis, it seems likely that the mode of infection may have been through the inhalation of oncospheres, completing the infective life cycle of the Echinococcus parasite. The rupture of cysts causes patients to become symptomatic and cyst rupture may occur as a consequence of trauma or secondary infection. Diagnosis is often dependant on a combination of serological and radiological testing. As demonstrated in this case, serology may only become positive as a result of cystic rupture. It is important to note that a negative serological test result does not exclude a diagnosis of cystic Echinococcus, particularly in cases of extra hepatic disease. Albendazole is the drug of choice for effective treatment of cystic Echinococcus, with no clear evidence of efficacy with the addition of praziquantel.

\section{Affiliations \\ Delaram Zahedi, Parisa Moori, Iqra Ashraf, Irfan Hafeez \\ East Lancashire Hospitals NHS Trust, Blackburn, UK.}

\section{Acknowledgments}

We would like to thank the Liverpool School of Tropical Medicine and the Royal Liverpool Hospital (both Liverpool, UK) for their advice regarding this case. 


\section{Conflict of interest}

D. Zahedi has nothing to disclose. P. Moori has nothing to disclose. I. Ashraf has nothing to disclose. I. Hafeez has nothing to disclose.

\section{References}

1. Eckert J, Deplazes P. Biological, epidemiological, and clinical aspects of echinococcosis, a zoonosis of increasing concern Clin Microbiol Rev 2005; 17: 107

2. Bagheri R, Haghi S, Amini M, et al. Pulmonary hydatid cyst: analysis of 1024 cases. Gen Thorac Cardiovasc Surg 2011; 59 : 105-109.

3. Moro P, Schantz PM. Echinococcosis: a review. Int J Infect Dis 2009; 13: 125-133.

4. Tuna S, Duymus TM, Yanik HS. Hydatid cyst of biceps brachii associated with peripheral neuropathy. Int J Surg Case Rep 2015; 8: 150-153.

5. Sehgal S, Mishra B, Thakur A, et al. Hydatid cyst of mediastinum. Indian J Med Microbiol 2008; 26: 80-81.

6. Sotiraki S, Himonas C, Korkoliakou P. Hydatidosisechinococcosis in Greece. Acta Trop 2003; 85: 197-201.

7. Pappas G, Kiriaze IJ, Falagas ME. Insights into infectious disease in the era of Hippocrates. Int J Infect Dis 2008; 12: 347-350.

8. Gottstein B, Reichen J. Hydatid lung disease (echinococcosis/ hydatidosis). Clin Chest Med 2002; 23: 397-408.

9. Petavy A, Deblock S, Walbaum S. Life-cycles of Echinococcus multilocularis in relation to human infection. J Parasitol 1991; 77: 133-137.

10. Romig T, Deplazes P, Jenkins D, et al. Ecology and life cycle patterns of Echinococcus species. Adv Parasitol 2017; 95 : 213-314.

11. Huang $M$, Zheng $H$. Primary alveolar echinococcosis (Echinococcus multilocularis) of the adrenal gland: report of two cases. Int J Infect Dis 2013; 17: E653-E655.

12. Sarkar M, Pathania R, Jhobta A, et al. Cystic pulmonary hydatidosis. Lung India 2016; 33: 179-191.

13. Hamouri S, Al Manasra AR, Daradkeh $\mathrm{H}$, et al. Pulmonary hydatidosis patterns and clinical outcomes. Australas Med J 2018; 11: 135-142.

14. Rakhshanpour A, Harandi MF, Moazezi SS, et al. Seroprevalence of human hydatidosis using ELISA method in Qom Province, Central Iran. Iran J Parasitol 2012; 7: 10-15.

15. Sedaghat F, Sadjjadi SM, Hosseini SV, et al. Evaluation of a Simple Dot-ELISA in comparison with countercurrent immunoelectrophoresis for diagnosis of human hydatidosis. Clin Lab 2011; 57: 201-205.
16. Machnicka B, Dziemian E, Rocki B, et al. Detection of Echinococcus multilocularis antigens in faeces by ELISA. Parasitol Res 2003; 91: 491-496.

17. Seres S, lovu A, Cozma V, et al. Comparative diagnostic value of PCR and ELISA methods in the diagnosis of Echinococcus granulosus infection. Intern J Mol Med 2009; 24: S69-S69.

18. Ito A, Okamoto $M$, Ishiguro $T$, et al. Short report: an imported case of cystic echinococcosis in Japan diagnosed by imaging and serology with confirmation of Echinococcus granulosus-speclfic DNA sequences. Am J Trop Med Hyg 1998; 58: 790-792.

19. Marrone G, Crino' F, Caruso S, et al. Multidisciplinary imaging of liver hydatidosis. World J Gastroenterol 2012; 18: 1438-1447.

20. Hajar A, Elkhattabi W, Jabri $\mathrm{H}$, et al. Radio-clinical profile of pulmonary hydatidosis. Eur Respir J 2017; 50: Suppl. 61, PA4135.

21. Taiana J, Schieppati E, Zorraquin V. Pulmonary Echinococcus - surgical treatment in 124 hydatid cysts. Dis Chest 1954; 26: 686-692.

22. Boudaya M, Mohamed J, Berraies A, et al. Brief original scientific report: a new surgical approach for the treatment of left pulmonary and hepatic hydatid disease. Surg Today 2014; 44: 1971-1974

23. Sokouti M, Golzari SEJ, Kayhan S, et al. Recurrence following pulmonary hydatid disease surgery. World J Surg 2014; 38: 266-266

24. Carpintero P, Kindelan J, Montero R, et al. Primary hydatidosis of the peripheral muscles: treatment with albendazole. Clin Infect Dis 1997; 24: 85-86.

25. Mohamed A, Yasawy M, Al Karawi M. Combined albendazole and praziquantel versus albendazole alone in the treatment of hydatid disease. Hepatogastroenterology 1998; 45: 1690-1694

26. Ricken FJ, Nell J, Gruener B, et al. Albendazole increases the inflammatory response and the amount of Em2-positive small particles of Echinococcus multilocularis (spems) in human hepatic alveolar echinococcosis lesions. PLoS Negl Trop Dis 2017; 11: e0005636. 